\title{
Role of miR-126a in regulation of expression of anti-apoptotic protein BCL2
}

\author{
L. Gulyaeva \\ Federal Research Centre "Fundamental and Translational Medicine", Novosibirsk, Russia \\ Novosibirsk State University, Novosibirsk, Russia \\ e-mail:lfgulyaeva@gmail.com
}

Key words: BCL2 expression, miR-126, tumor cells, target in vitro assay

Motivation and Aim: Anti-apoptotic B cell lymphoma 2 (BCL2) family members play a key role in the regulation of intrinsic apoptosis. In many types of cancer apoptosis is suppressed due to upregulation of these proteins through multiple mechanisms. MicroRNAs (miRs) may also control BCL2 gene expression at post-transcriptional level. Thus far 12 miRNAs have been validated to target BCL2 [1]. Using in silico approach we found that miR-126 host gene $E g f l 7$ has ESRs and DRE binding sites in promoter [2]. Then we performed experiments to measure miR-126 expression under xenobiotic exposure and validated its interaction with 3'UTR of $B C L 2$ target gene.

Methods and Algorithms: We analyzed ChIP-seq data obtained from the encode project (T47D and Ishikawa cells treated with estradiol, respectively GSM803539 and GSM803422) to identify miR-coding genes with ESRs binding sites in their promoter regions [3]. To study of gene expression we used human cell cultures MCF-7, breast and endometrial cancer tissues. For detection of miR and BCL2, Egfl7 expression the stem-loop RT-PCR and RT-PCR respectively were used. For luciferase reporter experiments, 293FT and HepG2 cells were transfected with the pmirGLO vector with the corresponding inserts (bcl2 3'UTR/bcl2 3'UTR mutated) and the corresponding mimic/miR-126-3p inhibitors $(+126 \mathrm{mim} /+126 \mathrm{inh})$.

Results: In breast cancer, BCL2 protein expression was associated with low tumor grade, ER positivity, and favorable prognosis. We have shown that BCL2 is more frequently overexpressed in endometrial cancer tissues than in normal adjoined tissues. To understand the mechanism of elevated expression of this protein in tumour cells we determined the miR126a expression, which may potentially interact with BCL2 mRNA. Since host gene Egfl7 of this miR has ESRs and DRE binding sites we incubated MCF7 cells with various doses of benzo(a)pyrene (BP). It was shown 2 fold increase of miR126a and Egfl7 expression under the BP exposure. At the same time, the expression level of BCL2 target gene decreased in 5 times. Moreover, the expression level of miR-126a in luminal B type of breast cancer is reduced by 3-4 times. These results confirmed the tumor suppressor feature of this miR which may regulate $B C L 2$ expression. To proof this suggestion we preformed luciferase reporter experiments and have shown that luciferase activity in 293FT cells transfected with the vector containing insertion of bcl 2 3'UTR and the corresponding mimic was reliably reduced by $20 \%$, whereas an increase in the signal by $55 \%$ was observed with the inhibitor. Similar results were obtained on HepG cells. Conclusion: The results showed that miR-126a may regulate BCL2 gene expression in breast and endometrial cancer. Besides, ligands of AhR capable activate the transcription of Egfl7 and miR-126 in MCF-7 cells, followed by inactivation of the BCL-2 gene expression, and may be considered as potential therapeutic agents in the treatment of these cancers. Acknowledgements: Supported by the RSF (project No. 15-15-30012).

\section{References}

1. Cui J. and Placzek W.J. (2018) Post-Transcriptional Regulation of Anti-Apoptotic BCL2 Family Members Int. J. Mol. Sci. 19:308.

2. Ovchinnikov V.Y., Antonets D.V., Gulyaeva L.F. (2018) The search of CAR, AhR, ESRs binding sites in promoters of intronic and intergenic microRNAs. J Bioinform Comput Biol. 16(1):1750029.

3. Korch C., Spillman M.A., Jackson T.A., Jacobsen B.M., Murphy S.K., Lessey B.A., Craig Jordan V., Bradford A.P. (2012) DNA profiling analysis of endometrial and ovarian cell lines reveals misidentification, redundancy and contamination. Gynecol Oncol. 127:241-248. 